\title{
Non-histone nuclear protein HMGN2 differently regulates the urothelium barrier function by altering expression of antimicrobial peptides and tight junction protein genes in UPEC J96-infected bladder epithelial cell monolayer
}

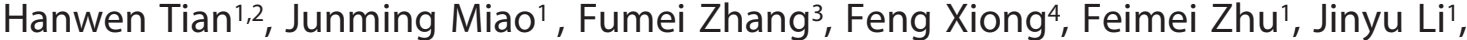 \\ Xiaoying Wang ${ }^{1}$, Shanzhe Chen ${ }^{1}$, Junli Chen ${ }^{1}$, Ning Huang ${ }^{1 凶}$ and Yi Wang ${ }^{1 凶}$
}

1Research Unit of Infection and Immunity, Department of Pathophysiology, West China College of Basic and Forensic Medicine, Sichuan University, Chengdu 610041, China; ${ }^{2}$ Department of Pharmacology, West China College of Basic and Forensic Medicine, Sichuan University, Chengdu 610041, China; ${ }^{3}$ Experimental Center, Northwest University for Nationalities, Lanzhou 730030, China; ${ }^{4}$ Department of Cardiology, The Third People's Hospital of Chengdu, Chengdu, China

The urinary tract is vulnerable to frequent challenges from environmental microflora. Uropathogenic Escherichia coli (UPEC) makes a major contribution to urinary tract infection (UTI). Previous studies have characterized positive roles of non-histone nuclear protein HMGN2 in lung epithelial innate immune response. In the study presented here, we found HMGN2 expression was upregulated in UPEC J96-infected urothelium. Surprisingly, over-expression of HMGN2 promoted disruption of BECs 5637 cells' intercellular junctions by down-regulating tight junction (TJs) components' expression and physical structure under $J 96$ infection. Further investigation showed that BECs 5637 monolayer, in which HMGN2 was over-expressed, had significantly increased permeability to J96. Our study systemically explored the regulatory roles of HMGN2 in BECs barrier function during UPEC infection and suggested different modulations of intracellular and paracellular routes through which UPEC invades the bladder epithelium.

Key words: HMGN2, UPEC, BECs 5637, bladder epithelium, tight junction, antimicrobial peptides

Received: 12 June, 2017; revised: 28 November, 2017; accepted: 26 December, 2017; available on-line: 17 March, 2018

e-mail: Ning Huang: huangpanxiao@sina.com; Yi Wang: wangyi83@scu.edu.cn

Abbreviations: HMGNs, high mobility group nucleosome-binding proteins; JAMs, junction-adhesion molecules; LPS, lipopolysaccharide; UPEC, uropathogenic Escherichia coli; UTI, urinary tract infection

\section{INTRODUCTION}

The urinary tract is subject to frequent challenges from the gut microflora and up to $60 \%$ of women will experience at least one time UTI during their lifetime (Hooton et al., 2004). UPEC makes a major contribution to UTI and they typically initiate these cases by invading the superficial epithelium that lines the bladder lumen (Foxman, 1990). The formation of a selectively permeable epithelial barrier is essential in preventing the uncontrolled passage of pathogens from the external environment to the internal tissue. Essential components of this epithelial barrier are physical intercellular structures termed tight junctions which are made by members of the Claudin family members, such as Occludin, the junction-adhesion molecules (JAMs) and other modulators
(Guttman \& Finlay, 2009). Besides these components, urothelia also participate in maintaining the intrinsic barrier function through lipopolysaccharide (LPS)-mediated TLR4 signaling (Schilling et al., 2003). As a result, a variety of secreted proteins involved in inflammatory responses, including IL-8, IL-6 (Billips et al., 2007), GMCSF (Li et al., 2017) and antibacterial mucosal proteins, such as the Surfactant Protein A (Hashimoto et al., 2017), are expressed. Therefore, like in the case of epithelium lining other parts of the body, invading pathogens can cross the urothelial barrier through intracellular tight and adherent junctions (paracellular route) or pass the apical surface of epithelial cells (transcellular route) (Balkovetz \& Katz, 2003).

High mobility group nucleosome-binding proteins (HMGNs) are small non-histone nuclear proteins associated with chromatin to modulate various nuclear functions (Hock et al., 2007). HMGN2 is one of the major members of HMGNs that is ubiquitously present in almost all embryonic and adult tissues of higher vertebrates. Previous study has shown the antimicrobial polypeptides (AMPs) function of HMGN2 is directed against fungi, bacteria and viruses (Ming et al., 2007). Recent studies have demonstrated critical roles of endogenous HMGN2 in alveolar epithelial cell innate immune responses against multiple bacterial pathogens and toxins (Deng et al., 2011; Liu et al., 2017; Teng et al., 2016; Wang et al., 2016). Specifically, HMGN2 has been reported to activate $\mathrm{NF}-x \mathrm{~B}$ signaling and promote LPS-induced $\beta$-defensin expression in A549 cells (Deng et al., 2011). Additionally, HMGN2 is found to be involved in the regulation of Psendomonas aeruginosa (PA) toxin Pyocyanin (PCN)-induced epithelial cell oxidative stress by promoting intracellular ROS clearance and attenuating PA invasion (Liu et al., 2017). However, how HMGN2 influences physical intercellular structure and innate immune function in the context of epithelium barrier has not been clarified yet. In the study presented here, we found HMGN2 expression was up-regulated in the UPEC J96-infected urothelium. Re-analysis of the transcription profile of LPS/si-HMGN2 double-treated lung A549 cells revealed opposing effects on AMP gene expression and TJs function, which were in line with the transcription alternation in J96-infected BECs 5637. Surprisingly, over-expression of HMGN2 promoted disruption of BECs 5637 intercellular junction by down-regulating the TJs protein expression and physical structure 
under J96 infection. Further investigation showed that in BECs 5637 monolayer, where HMGN2 was over-expressed, there was a significantly increased permeability to invading bacteria. Our study systemically explored the regulatory roles of $\mathrm{HMGN} 2$ in BECs barrier function during UPEC infection and suggested diversified modulations on intracellular and paracellular routes through which UPEC invades the bladder epithelium.

\section{MATERIALS AND METHODS}

Materials. The following antibodies were used: rabbit polyclonal HMGN2 antibody (Cell Signalling Technology), mouse monoclonal GAPDH antibody (Beyotime Biotechnology), Rabbit monoclonal Occludin antibody (invitrogen, Thermofisher scientific), ZO-1 (invitrogen, Thermofisher scientific), E-cadherin (Cell Signalling Technology). The plasmids used in this study came from our Lab collection. The siRNAs were purchased from RiboBio Co., Ltd.

Cell line and cell culture. Human bladder epithelial 5637 cells were obtained from the Cell Bank of Chinese Academy of Sciences. These cells were cultured in RPMI Medium 1640 supplemented with 10\% fetal bovine serum (FBS) at $37^{\circ} \mathrm{C}$ in a humidified incubator containing $5 \% \mathrm{CO}_{2}$.

Stable cell line construction. 5637 cells were seeded in 12-well plates at a density of $2 \times 10^{5}$ cells per well. Following overnight incubation in the RPMI Medium 1640 containing $10 \% \mathrm{FBS}$, at $37 \%$ and $5 \% \mathrm{CO}_{2}$, the pEx-HMGN2 and pEx-NC plasmids were transfected into 5637 cells using Lipofectamine 2000 (Invitrogen, Thermofisher scientific) according to the manufacturer's instructions. Resistant colonies were selected by G418 at $400 \mu \mathrm{g} / \mathrm{mL}$ based on the killing curve patterns for 5637 cells. Single clones were picked and tested for HMGN2 expression by RT-PCR and western blot.

Animal infection model establishment. Adult female C57 mice (8 to 10 weeks of age) were anesthetized by intraperitoneal injection of $2 \%$ chloral hydrate $(15 \mu \mathrm{l} / \mathrm{g}$ body weight and inoculated via transurethral catheterization with $10^{8}$ colony-Forming Units (CFU)) of viable J96 in $100 \mu$ l of PBS for $24 \mathrm{~h}$. The study was approved by the medical management division of West China College of Preclinical Sciences and Forensic Medicine, Sichuan University, Chengdu, China.

Immunofluorescence staining. BECs 5637 stably expressing GFP-vector pEx-NC) and GFP-HMGN2 (pEX-HMGN2) were seeded on a coverslip and fixed in $4 \%$ PFA for $30 \mathrm{mins}$ and then permeabilized $(0.1 \%$ TritonX-100) for 30 mins at room temperature. After being blocked in the blocking buffer $(5 \%$ BSA) for $1 \mathrm{~h}$, the cells were incubated overnight with primary antibodies, followed by $2 \mathrm{~h}$ incubation with fluorescently-conjugated secondary antibodies at $37^{\circ} \mathrm{C}$. The stained cells were visualized by FV1000 confocal microscope (Olympus).

siRNA transfection. BECs5637 cells were plated in 6 -well plates (Costar) at a density of $5 \times 10^{5}$ cells per well. Cells were grown to $70 \%$ confluence and transfected by si-HMGN2 and si-NC using Lipfectamine 2000 (Invitrogen, Thermofisher scientific), according to the manufacturer's instructions. Human HMGN2-specific small RNA (siRNA-HMGN2, sense: 5'-AGU CAG CGG CUU-3', antisense: 5'-UCA CAA GCC GAC CCU-3') and negative control siRNA (siRNA-NC) were synthesized by RiboBio Co.Ltd. The medium was replaced $6 \mathrm{~h}$ after transfection and the cells were cultured for $24 \mathrm{~h}$ before the efficiency of HMGN2 knockdown was determined.
RNA isolation, RT-PCR and real time PCR. Total RNA was isolated from BECs 5637 cells using Trizol (Invitrogen, Thermofisher scientific) following the manufacturer's instructions. cDNA was synthesized using All-in-One cDNA Synthesis SuperMix (Bimake). The harvested cDNA was analyzed by SYBR Green PCR kit according to the manufacturers' protocols. 5 $\mu \mathrm{L}$ cDNA was amplified in a $25 \mu \mathrm{L}$ reaction mix containing $22.5 \mu \mathrm{L}$ SYBR Green supermix (Bio-Rad) and $1 \mu \mathrm{L}$ each primer. The amplification conditions were as follows: initial denaturation at $95^{\circ} \mathrm{C}$ for $15 \mathrm{~min}, 35$ cycles of denaturation at $95^{\circ} \mathrm{C}$ for $15 \mathrm{~s}$, annealing at corresponding temperature for $15 \mathrm{~s}$ and elongation at $72^{\circ} \mathrm{C}$ for $30 \mathrm{~s}$. The experiment was repeated three times under each experimental condition and the comparative threshold cycle method was employed with human GAPDH as the internal control. Analysis of relative gene expression data was done by using realtime quantitative PCR and the $2^{-\Delta \Delta c t}$ method. Primers for individual mRNAs were synthesized by TsingKe Biological Technology: HMGN2 sense, CCATTGAAGAAGGGAGTTTGA; antisense, ATCAGAGGCAGCATTCCAAG. HBD1-sense, ATGAGAACTTCCTACCTTCTGCT; antisense, TCTGTAACAGGTGCCTTGAATT'T. HBD2-sense, CTCCTCT'TCTCGTTCCTCTTCA; antisense, GCAGGTAACAGGATCGCCTAT. HBD3-sense, TCCAGGTCATGGAGGAATCAT; antisense, CGAGCACTTGCCGATCTGT. Psoriasin-sense, ACGTGATGACAAGATTGACAAGC; antisense, GCGAGGTAATT'TGTGCCCT'T'T. RNAse 7-sense, CGTGTCCCTGACCATGTGTAA; antisense, GACTTGTTCTGTCGCTTCTCTT. Cathelicidin-sense, AGGTCCTCAGCTACAAGGAAG; antisense, TCTTGAAGTCACAATCCTCTGGT. E-cadherin-sense, ACCTCTGTGATGGAGGTC; antisense, CCACATTCGTCACTGCTACG. Occludin-sense, TGCATGTTCGACCAATGC; antisense, AAGCCACTTCCTCCATAAGG. ZO-1-sense, ACCATCATTGTCGTCGCATGTAGATCC; antisense, GATGCTCTAGGTGCCTGTTCGTAACG. CLDN1-sense, GCGCGATATT'TCT'TCT'TGCAGG; antisense, TTCGTACCTGGCATTGACTGG. GAPDH-sense, TGCACCACCAACTGCTTAGC; antise nse,GGCATGGACTGTGGTCATGAG.

cDNA microarray and data analysis. Microarray assay was done as described previously (Deng et al., 2011). Briefly, A549 cells were placed into six-well plates at a density of $2 \times 10^{5}$ cells per well. The cells were divided into four groups in triplicate: WT group (untreated A549), HMGN2 ${ }^{\text {kd }}$ (A549 cells transfected with HMGN2-specific siRNA2), WT+LPS group (A549 cells treated with 100 $\mu \mathrm{g} \cdot \mathrm{mL}^{-1} \mathrm{LPS}$ ) and HMGN2 $2^{\mathrm{kd}}+\mathrm{LPS}$ group (A549 cells transfected with HMGN2-specific siRNA2 for $48 \mathrm{~h}$ and then treated with $100 \mu \mathrm{g} \cdot \mathrm{mL}^{-1} \mathrm{LPS}$ for $24 \mathrm{~h}$ ). The microarray was performed at Shanghai Biochip according to instructions from SBC GeneChips (shbiochip, Shanghai). Total RNA was isolated from A549 cells using Trizol (Invitrogen) following the manufacturer's instructions. About $10-20 \mu \mathrm{g}$ of RNA were converted to cDNA using an oligo (dT) T7 primer, and the double-stranded cDNA was labelled with $\mathrm{Cy} 3$ (as the reference sample) and Cy5 (as the treatment sample), respectively. The samples were hybridized competitively under coverslips to the microarray slides at $50^{\circ} \mathrm{C}$ for $16 \mathrm{~h}$ in a dark humidity chamber and washed as follows: $2 \times \mathrm{NaCl} / \mathrm{Cit}$ for $5 \mathrm{~min}, 0.5 \times \mathrm{NaCl} / \mathrm{Cit}$ for $5 \mathrm{~min}$, and $30 \%$ ethanol/water for $30 \mathrm{~s}$ at $42^{\circ} \mathrm{C}$. Hybridization experiments were performed in duplicate using cDNA derived from the four different groups. Three biological replicates (independent RNA isolations), each with a technical replicate (dye 


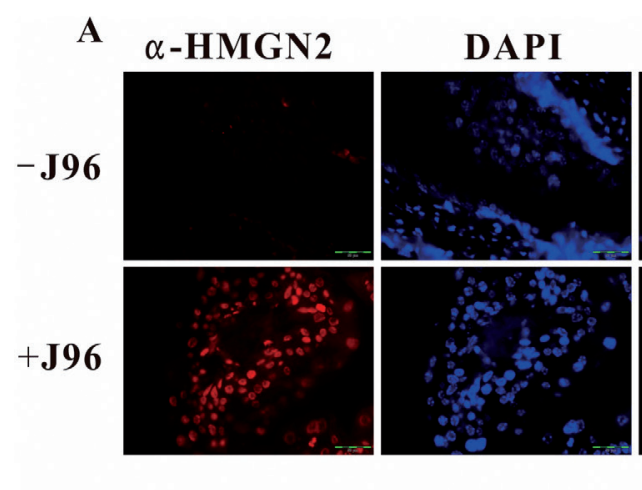

C

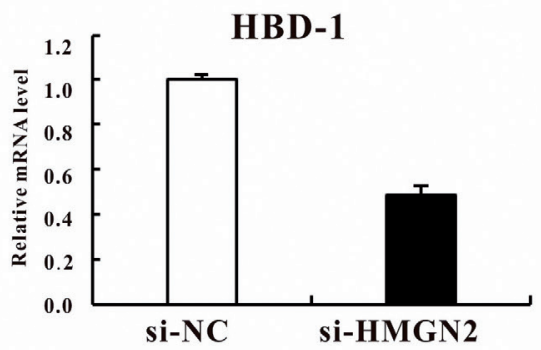

E

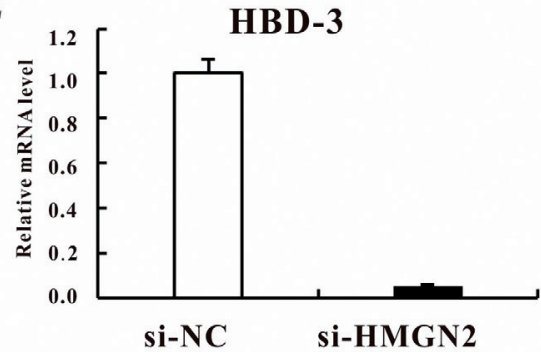

G

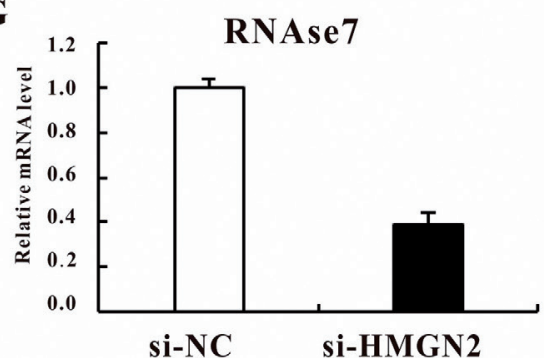

Merge

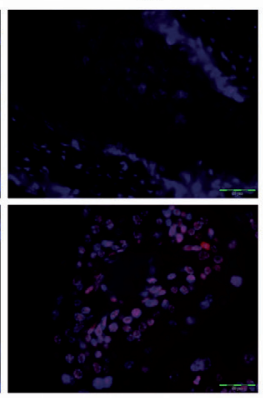

D

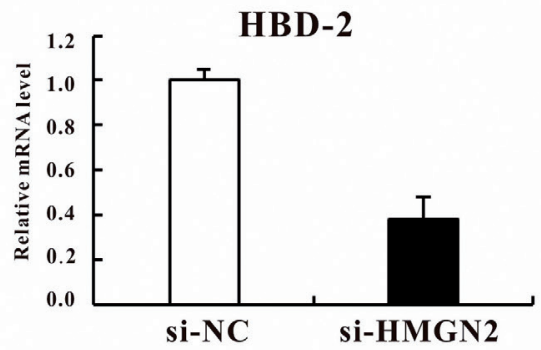

$\mathbf{F}$

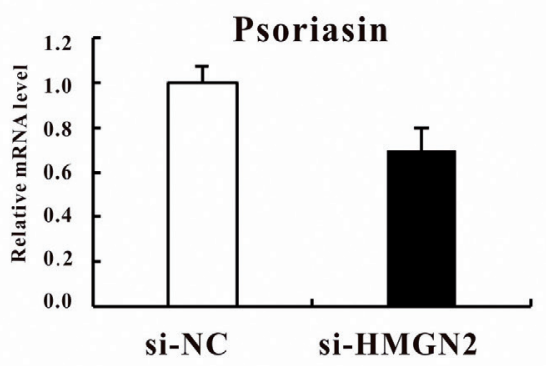

H

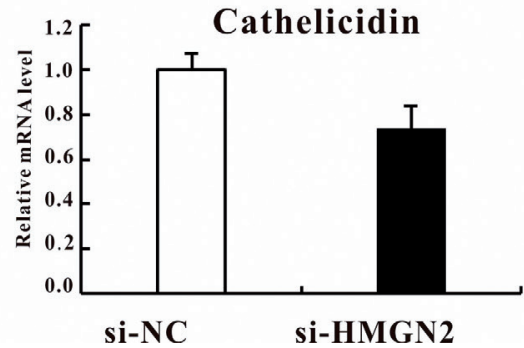

Figure 1. HMGN2 facilitates AMPs gene expression in UPEC-infected BECs 5637.

(A) Elevated HMGN2 expression in UPEC J96-infected mice bladder epithelia. Mice bladders were administrated with UPEC J96 (108 CFU) by transurethral catheterization. Then the immuno-fluorescence assay was applied using anti-HMGN2 antibody (a-HMGN2, red) and DAPI (blue). Confocal microscopy images were acquired. Bars: $50 \mu \mathrm{m}$. (B) Western blotting showing the knockdown efficiency of siRNA oligo targeting HMGN2 under UPEC J96 (MOI=100:1) infection. (C-H) mRNA levels of AMPs genes affected by HMGN2 knockdown in J96-infected 5637 cells. (Results presented relative to GAPDH and expressed as the mean \pm S.D. $(n=3)$ ).

swap), were performed for each condition. Genes with a Cy5/Cy3 signal ratio $\geq 2.0$ were considered upregulated, whereas those with a ratio $\leq 0.5$ were down-regulated. Western blot. Whole cell lysate was extracted from BECs 5637 cells using Protein Extraction Reagent (Beyotime Biotechnology) following the manufacturer's instructions. The protein concentration of the extract was determined with the BCA protein assay (Pierce). The proteins were separated with SDS/PAGE and transferred to polyvinylidene difluoride membranes. The membranes were incubated with HMGN2, ZO-1, Ecadherin, Occludin and GAPDH antibodies followed by incubation with the horseradish peroxidase conjugated anti-goat IgG serum. Finally, the membranes were developed using the ECL Plus reagent (Pierce).

Paracellular flux assays. Flux of fluorescein isothiocyanate-labeled dextran (molecular weight, 3kD; Sigma-Aldrich) across BECs 5637 monolayer was assayed. Briefly, monolayers were washed free of media and transferred to phosphate buffer saline. The apical chamber was gently aspirated and replaced with $50 \mu \mathrm{L}$ of 1 $\mathrm{mg} / \mathrm{ml}$ of fluorescein isothiocyanate-dextran $(1 \mathrm{mg} / \mathrm{ml})$. The monolayers were rotated on an orbital shaker (60 $\mathrm{rpm})$ at $37^{\circ} \mathrm{C}$, and samples $(50 \mu \mathrm{L})$ were removed from the basal chamber after 60 minutes. Fluorescence of these samples was determined using a fluorescent plate 
Table 1. Microarray profile of A549 cells treated with LPS and/or si-HMGN2 siRNA oligo

(Genes discussed in the main text are highlighted, \# represents TJs-associated genes, *represents AMPs-associated genes).

\begin{tabular}{|c|c|c|c|c|c|}
\hline \multirow[b]{2}{*}{ GenBank } & \multirow[b]{2}{*}{ Entrez_Gene_Name } & \multirow[b]{2}{*}{ Entrez_Gene_Description } & \multicolumn{3}{|c|}{$\log 2$ Ratio } \\
\hline & & & $\begin{array}{c}\text { WT+LPS } \\
\text { /WT }\end{array}$ & $\begin{array}{c}\text { HMGN2 }^{\mathrm{KD}+L P S} \\
/ \text { WT+LPS }\end{array}$ & $\begin{array}{c}\text { HMGN2 }^{\mathrm{KD}} \\
\text { /WT }\end{array}$ \\
\hline NM_004942.2 & DEFB4 * & HBD-2 & 5.2761953 & -3.227745 & -3.446539 \\
\hline NM_018661.2 & DEFB103A * & HBD-3 & 4.169443 & -2.6733924 & -3.193863 \\
\hline NM_000584.2 & IL-8 & interleukin 8 & 3.915317 & -1.070854 & -1.969843 \\
\hline NM_182848.2 & CLDN10 \# & claudin 10 & 3.27054019 & 1.1713224 & 4.42582719 \\
\hline NM_021101.3 & CLDN1 \# & claudin 1 & 0.887185 & 1.168331 & 1.508518 \\
\hline NM_002538.2 & OCLN \# & occludin & 0.4902146 & 0.3122318 & 2.9555476 \\
\hline BG696638 & DEFB1 * & HBD-1 & 0.4305803 & -1.2047543 & -0.08439 \\
\hline NM_004345.3 & CAMP* & $\begin{array}{c}\text { cathelicidin } \\
\text { antimicrobial peptide }\end{array}$ & 0.066878 & -0.2231013 & -0.522334 \\
\hline AK023343 & RNASE7 * & $\begin{array}{c}\text { ribonuclease, RNase A } \\
\text { family, } 7\end{array}$ & -0.0120735 & -2.1366295 & -0.740169 \\
\hline BQ051103 & HMGN2 & $\begin{array}{c}\text { high-mobility group } \\
\text { nucleosomal binding } \\
\text { domain } 2\end{array}$ & -0.2945625 & -7.896049 & -8.5550325 \\
\hline BG680212 & S100A7 * & $\begin{array}{l}\text { S100 calcium binding } \\
\text { protein A7 (psoriasin ) }\end{array}$ & -0.33581 & -0.395155 & -0.8731623 \\
\hline NM_001307.3 & CLDN7 \# & claudin 7 & -0.338948 & 0.827961 & 0.353449 \\
\hline NM_003257.2 & TJP1 \# & zona occludens 1 & -0.6272255 & 0.304018 & -0.664593 \\
\hline
\end{tabular}

reader (Thermofisher scientific). Molar flux was calculated from a standard curve that was prepared daily.

Flat colony counting method. BECs 5637 cells were exposed to UPEC J96 until the monolayer was formed, then cells were infected with UPEC J96 (MOI=10, 50, 100) for 2 hours. $100 \mu \mathrm{L}$ sample from the lower chamber was taken and plated onto LB agar plates. Colonies were counted to quantify the number of bacteria that crossed the monolayer.

Statistical analysis. Data were expressed as mean values \pm standard deviation (S.D.). All data analysis was tested by one-way analysis of variance for multiple comparisons with the LSD-test (homogeneity of variance) and Tamhane's T2-test (heterogeneity of variance).

\section{RESULTS}

HMGN2 alters transcription profile of innate immune response genes in LPS-stimulated lung epithelial cells

Recently, we found that the expression of HMGN2 was elevated in K. pneumoniae infected A549 and HBE 16 cells (Teng et al., 2016). To investigate whether HMGN2 is associated with UPEC infection in BECs, an acute bacterial cystitis in mice model was established by transurethral infection of UPEC J96 strain. Immunofluorescence staining showed that the expression of HMGN2 was obviously increased in BECs from J96-infected mice (Fig. 1A) Our previous study displayed a genome-wide gene expression profile in LPS-treated and/or HMGN2 down-regulated A549 cells and found several genes involved in innate immune response were substantially affected (Deng et al., 2011). Most of these genes are involved in NF- $x$ B pathway-mediated cytokines and AMPs such as human defensin $\beta-2$ (HBD-2) and human defen$\sin \beta-103 \mathrm{~A}$ (HBD-3). Re-analysis of this profile revealed that besides HBD-2 and HBD-3, mRNA levels of other major AMP genes encoding HBD-1, Cathelicidin, Psoriasin and RNAse7 were also inhibited by HMGN2 knockdown in LPS-treated A549 cells (Table 1, HMGN2 ${ }^{\mathrm{kd}}$ +LPS/WT+LPS, marked by asterisks). Surprisingly, contrary to expression changes of AMP genes and other innate immunity-associated genes including IL-8, IL-10, as well as Mucin family members, the microarray data showed opposing effects of HMGN2 knockdown on the expression of genes encoding major TJs proteins. As the results show, mRNA levels of Claudin 1, Claudin 10, Claudin 7 and Occludin were promoted by HMGN2 knockdown in both, LPS-treated and untreated cells, while zona occludens $1(\mathrm{ZO}-1)$ and E-cadherin moderately increased in LPS-treated/HMGN2 knockdown cells (Table 1, HMGN2 ${ }^{\mathrm{kd}}+\mathrm{LPS} / \mathrm{WT}+\mathrm{LPS}$ and HMGN2 ${ }^{\mathrm{kd}}$ +LPS/WT, marked by pound). To this end, it suggests that there would be different regulation of HMGN2 in the bladder epithelial barrier function.

\section{HMGN2 knockdown decreases mRNA levels of AMP genes in J96-infected BECs 5637}

To further investigate how HMGN2 regulates bladder epithelial function during UPEC infection, we firstly conducted RT-qPCR assay to check mRNA lev- 


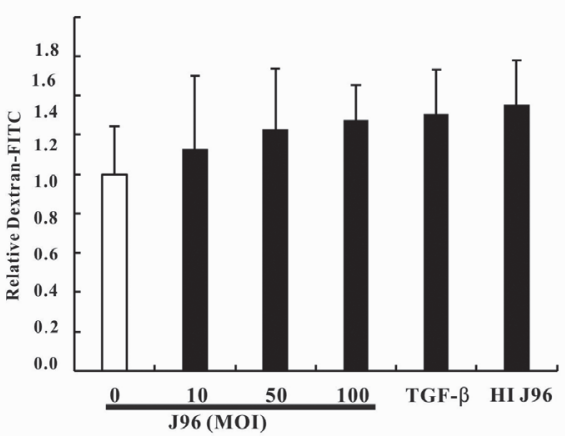

C

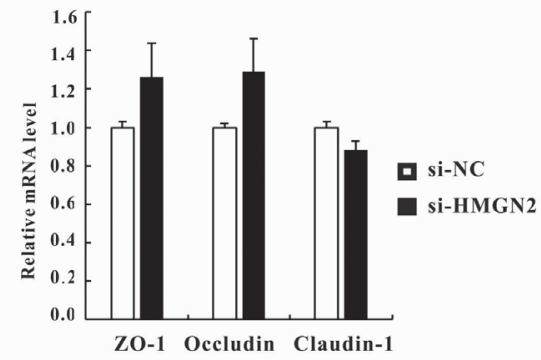

$\mathbf{E}$

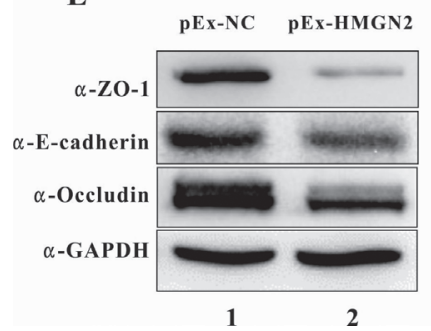

B

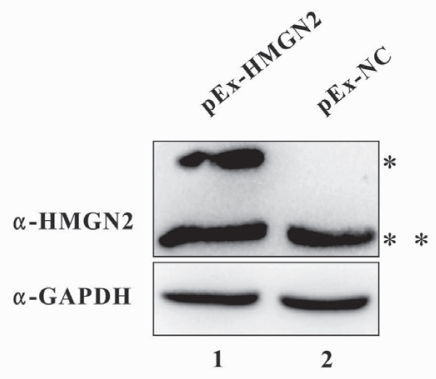

D

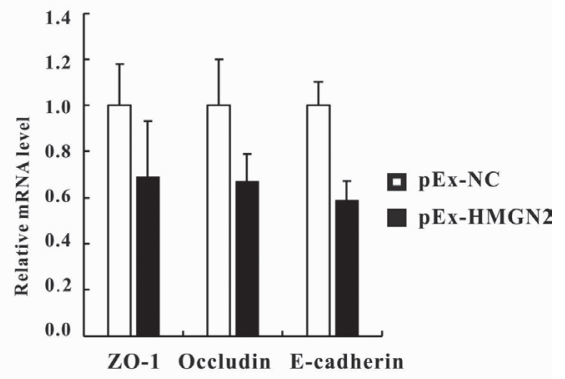

Figure 2. HMGN2 down-regulates barrier function and inhibits TJs-associated gene expression in J96-infected BECs 5637 monolayer. (A) BECs 5637 confluent monolayer stably over-expressing HMGN2 was exposed to increasing doses of UPEC J96 (MOI=0,10,50, 100) or heat inactivated UPEC J96 (HI J96 MOl=100:1) for 2 hours or to TGF- $\beta(2 \mathrm{ng} / \mathrm{ml})$ for 24 hours respectively. The relative Dextran permeability was calculated by dividing fluorescence density of HMGN2 overexpression group (pEx-HMGN2) by control group (pEx-NC). (B) Western blotting showing the over-expression of GFP- HMGN2 (a-HMGN2, marked by single asterisk; double asterisk indicates endogenous HMGN2). (C-E) BECs 5637 transfected with si-HMGN2 or pEX-HMGN2 followed by 2-hour UPEC J96(MOI=100:1) infection. siRNA-NC or pEX-NC was used as control. (C-D) mRNA levels and (E) protein levels of TJs-associated genes after treatments (Results presented relative to GAPDH and expressed as the mean \pm S.D. $(n=3)$ ).

els of AMP genes in J96-infected BECs 5637 with or without down-regulated HMGN2 expression. Synthetic siRNA oligo targeting 3'UTR of HMGN2 substantially reduced HMGN2 protein level in BECs 5637 (Fig. 1B). Consistently, we observed dramatic decrease in AMP gene expression in HMGN2 knockdown cells when compared to PBS-treated counterparts (Fig. 1 $\mathrm{C}-\mathrm{H})$. Among these genes, most significant drop of expression $(90 \%)$ was shown for HBD-3, while the mildest inhibition (about 30\%) for genes encoding Psoriasin and Cathelicidin. In addition, the inhibition on HBD-1, HBD2 and RNAse 7 expression was up to around $50 \%$. Therefore, HMGN2 confers similar impacts on AMP genes in BECs 5637 as that found in the A549 cells.

\section{HMGN2 down-regulates barrier function and inhibits TJs-associated gene expression in J96-infected BECs 5637 monolayer}

Our data imply different HMGN2 regulation of the AMP and TJ genes, which are involved in the host defense against transcellular and paracellular invasion of pathogens, respectively (Balkovetz \& Katz, 2003). We therefore conducted transwell assay to measure paracellular permeability of BECs 5637 to FITC-labelled Dextran, trying to dissect the impact of HMGN2 on each individual pathway. As shown by our data, incubating confluent BECs 5637 monolayer with different MOI of J96 for 2 hours elevated the relative permeability to Dextran between the HMGN2 overexpression group and the vehicle group (Fig. 2A). Such effect in the J96-infection group was comparable to that of TGF- $\beta$ treated one, where the epithelium barrier function has been shown to be obviously disrupted (Overgaard et al., 2015). Of note, incubation with heat-inactivated J96 (HI J96) also caused a dramatic increase in Dextran permeability in the HMGN2 over-expressed BECs 5637 monolayer, suggesting the dysfunction of confluent monolayer was not due to colonization by bacteria. Thus, although HMGN2 has dual functions in facilitating AMPs expression and potential inhibition of TJs, it is more likely that during bacterial infection, the paracellular path would be a more vulnerable target attacked by invading bacterial pathogen through the HMGN2-mediated TJs dysfunction.

To prove our hypothesis, we further investigated the major 'TJs' expression in J96-infected BECs 5637 cells 
pEx-NC

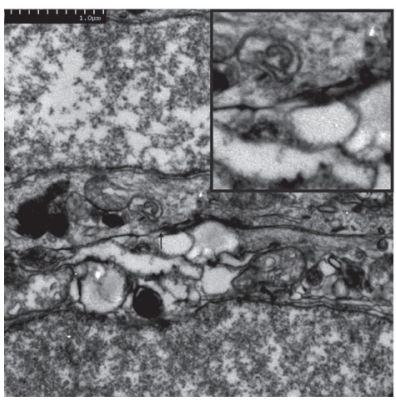

pEX-HMGN2

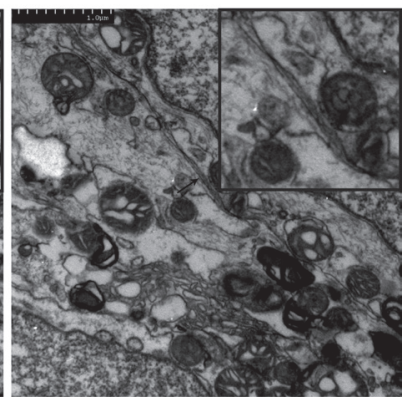

Figure 3. HMNG2 promotes $J 96$ infection-induced TJs disruption in BECs 5637 cells.

Transmission electron microscope (TEM) image of BECs 5637 confluent monolayer infected with UPEC $J 96$ (MOl=100:1) for 2 hours. TJs are indicated by black arrows and details are shown in enlarged images (right upper corner).

where HMGN2 was transiently repressed (si-HMGN2) or stably over-expressed (pEx-HMGN2). As shown in Fig. 2B, GFP-tagged HMGN2 (marked by a single asterisk) was steadily expressed along with endogenous HMGN2 (marked by a double asterisk), indicating the success of HMGN2 overexpression. In HMGN2 knockdown cells, increased ZO-1 and Occludin mRNA levels and relatively intact Claudin-1 mRNA level were seen (Fig. 2C), while reduced mRNA levels of ZO1, Occludin and E-cadherin were shown in HMGN2 over-expressed cells (Fig. 2D). Moreover, Western blotting confirmed findings of the RT-qPCR experiments by indicating a significant decrease of ZO-1, Occludin and E-cadherin protein levels in BECs 5637 under J96 infection (Fig. 2E).

\section{HMNG2 promotes J96 infection-induced TJs' disruption in BECs 5637 cells}

To investigate the morphological alternation of BECs 5637 TJs, the ultrastructure of paracellular regions of J96-infected cells, with or without over-expressed HMGN2, were analyzed by transmission electron microcopy (TEM). The confluent monolayer of BECs 5637 transfected with either empty vector (pEx-NC) or HMGN2 over-expression ( $\mathrm{pEx}-\mathrm{HMGN}$ ) vector were infected with 100:1 MOI of J96 for 2 hours. Compared with empty vector group, HMGN2 over-expressed group

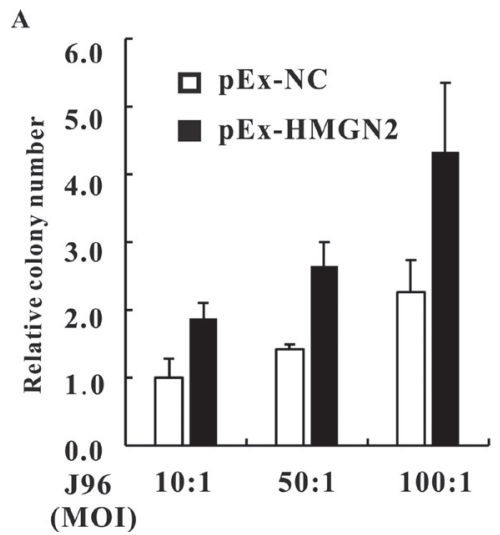

presented reduced electron-dense material between cells and enlarged intracellular space (Fig. 3 right, indicated by an arrow indicated and detailed in enlarged image). These results demonstrate that HMGN2 promotes disruption of TJs' ultra structure during J96-infection.

\section{HMGN2 overexpression enhanced the disruption of BECs 5637 monolayer TJs during $J 96$ infection}

Epithelium barrier function is composed of combinatorial effects of intrinsic structural integrity which is maintained by cell-cell connecting molecules and extrinsic mucosal immunity system, including secretion of mucins, complements and AMPs (Pitman \& Blumberg, 2000). The result of Dextran permeability assay (Fig. 2A) and TEM (Fig. 3) analysis indicated that HMGN2 facilitates the dysfunction of paracellular defense by directly compromising TJs' ultrastructure, while it also elevates intracellular innate immune response, including $\mathrm{NF}-x \mathrm{~B}$ signaling (Deng et al., 2011) and AMPs gene expression (Fig. 1). We therefore sought to systematically study the impact of HMGN2 on BECs barrier function during bacterial infection. Confluent monolayer of 5637 cells stably transfected with empty vector ( $\mathrm{pEx}-\mathrm{NC}$ ) or HMGN2 over-expression (pEx-HMGN2) vector were exposed to increasing MOI of $\mathrm{J} 96$ for 2 hours in the upper chamber of transwell apparatus and the lower chamber fluid was then collected and spread on plates. The colonies were counted to measure the bacterial permeability of infected monolayer. Our results showed that when compared with the empty vector group, in HMGN2 over-expressed monolayer significantly increased bacterial invasion rates were observed (Fig. 4). Thus, despite opposing HMGN2 regulation of AMPs and TJs expression in BECs 5637, the overall effect during bacterial infection on cell monolayer barrier would be to promote UPEC invasion by disrupting intracellular TJs.

\section{DISCUSSION}

HMGN2 has been demonstrated to play multiple roles in lung epithelium innate immune response against various bacterial pathogens. Our previous study showed the intrinsic inhibitory effect of HMGN2 on actin skeleton dynamics regulation, which could be activated to serve as part of innate defense mechanism against $K$. pneumoniae invasion (Teng et al., 2016). Moreover, HMGN2 has been reported to

B

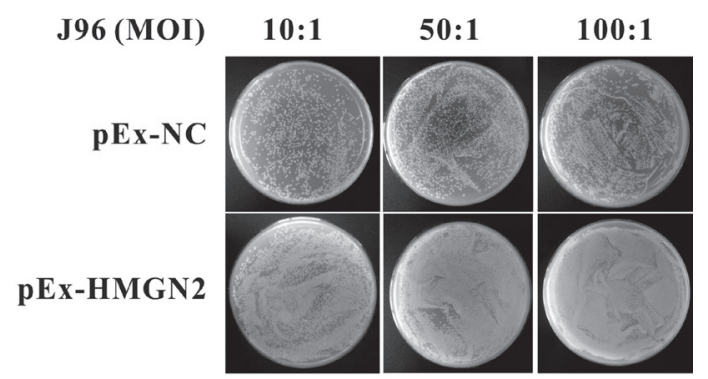

Figure 4. HMGN2 overexpression enhanced the disruption of BECs 5637 monolayer TJ during J96 infection.

BECs5637 stably expressing GFP-HMGN2 or vehicle control were exposed to increasing doses of UPEC J96 (MOI=10, 50,100 ) for 2 hours in the apical chamber of transwell apparatus. $100 \mu \mathrm{l}$ of the medium from lower chamber were taken for Flat colony counting. Results presenting relative colony number between HMGN2 overexpression (pEx-HMGN2) and vehicle control (pEx-NC) (A) and representative plates are shown (B) (Results expressed as the mean \pm S.D. $(n=3)$ ). 


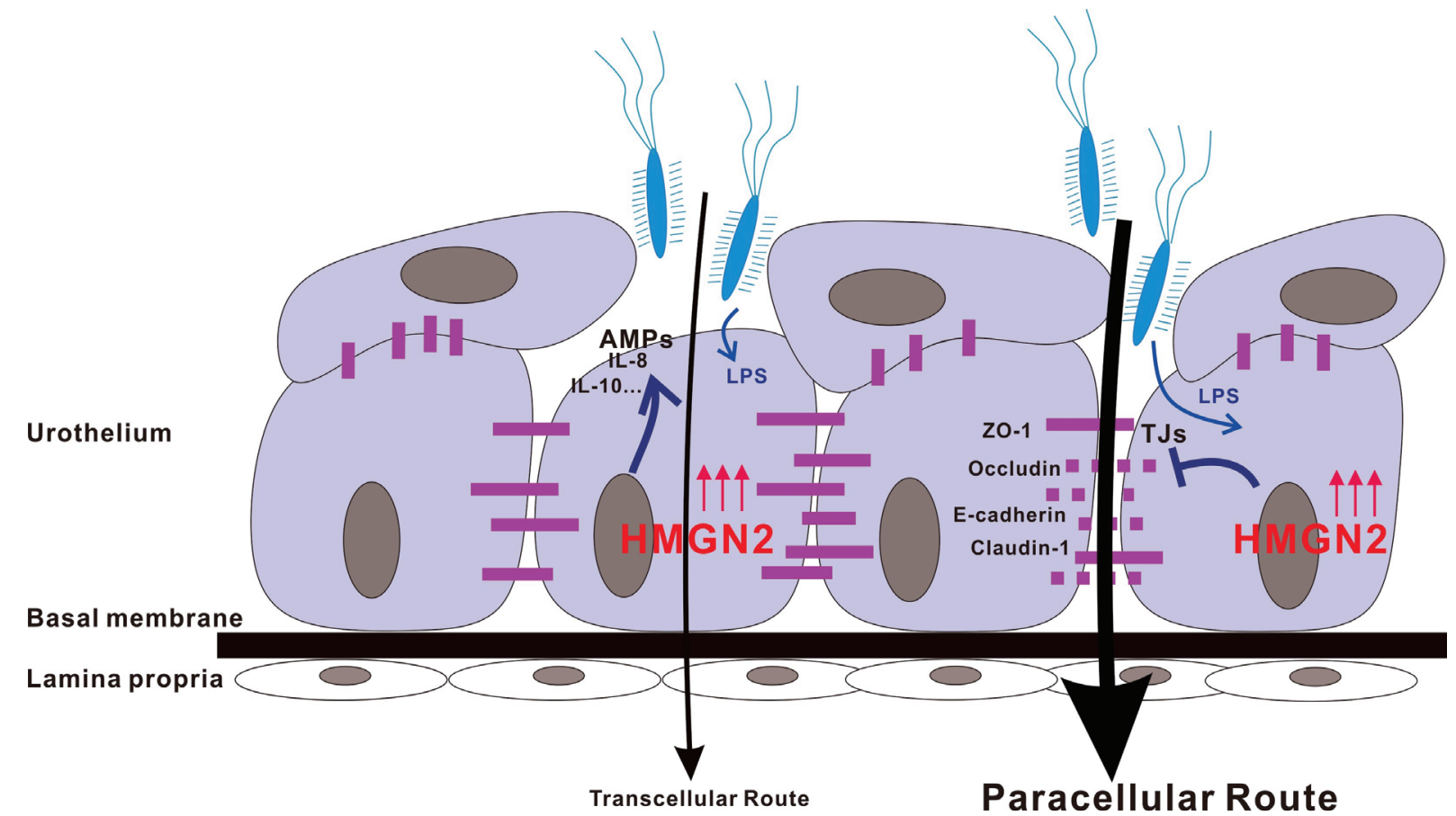

Figure 5. A schematic diagram depicting dual functions of HMGN2 on the transcellular and paracellular route of UPEC invasion in Urothelium.

LPS and other bacterial toxins activate HMGN2 expression, which in turn elevates expression of general innate immune response genes, such as IL-8, IL-10, and AMPs gene. Meanwhile increased HMGN2 represses expression of TJs proteins such as ZO-1, Occludin, E-Cadherin and Claudin-1 to disrupt paracellular TJs' ultrastructure and permeability. Thus, invading bacteria may pass through paracellular routes by up-regulating host HMGN2 expression in urothelium.

activate NF- $x \mathrm{~B}$ signaling and promote LPS-induced $\beta$-defensin expression (Deng et al., 2011) and to attenuate PA invasion by inhibiting cellular oxidative stress in A549 cells (Liu et al., 2017). In the study presented here, we found a similar HMGN2 regulation of different epithelium cells, including promotion of AMPs, IL-10 and IL-8 gene expression (Fig. 1 and Table 1). However, all of the above studies were performed to present a situation where an individual cell but not the whole lining epithelial barrier was challenged. Therefore, a study focusing on the barrier function of the epithelial surface was needed. Unexpectedly, our results showed the HMGN2 expression was elevated in J96-infected bladder epithelium, while over-expressed HMGN2 could down-regulate major TJs proteins, ZO-1, E-cadherin and Occludin expression at the mRNA and protein levels (Fig. 2). In line with this finding, overexpression of HMGN2 caused disruption of ultrastructure of intracellular barrier (Fig. 4). Moreover, increased BECs 5637 monolayer permeability to Dextran under J96 infection (Fig. 2A) was shown, supporting the notion that disruption of paracelluar physical barrier contributes to impaired monolayer integrity, as non-microbial reagent Dextran was less likely to pass through apical sides of epithelium in a relative short time frame ( 2 hours). Lastly, we checked the overall effect of HMGN2 on BECs monolayer barrier function against J96, and our result showed that HMGN2 facilitates J96 invasion. Despite positive effects of HMGN2 on intracellular innate immune response, we propose that the increased J96 invasion rate resulted from disrupted paracellular physical barrier (Fig. 5).

Previously, there was no systemic study investigating effects of HMGN2 in the context of the epithelium barrier. HMGN2 regulates innate immune response to dampen bacterial pathogen invasion by regulating either actin polymerization or ROS clearance which function in the transcellular route (Liu et al., 2017). In the study presented here, HMGN2 was shown to give a dual regulation in both, the intracellular innate immune response (such as AMPs expression), and the paracellular physical barrier maintenance responding to the intruding bacteria (Fig. 5). It is legitimate to postulate that a pathogenic toxin, such as LPS, activates HMGN2 through TLR4 and NF-xB signaling (Wu et al., 2017). Possible mechanisms by which HMGN2 in turn regulates other innate immune response genes, including TJs, would be: 1) a direct regulation of HMGN2 of TJs gene transcriptions. As a non-histone nuclear protein, HMGN2 interacts with nucleosome and other chromatin-associated proteins (Hock et al., 2007) and mainly enriches at promoter regions characterized by hypersensitivity to DNase I (Deng et al., 2015). It has been demonstrated that HMGN2 participates in gene transcription regulation by altering chromatin organization. For instance, HMGN2 accompanied HMGN1 to modulate chromatin environment in order to regulate gene expression during activation of naive $B$ cells (Zhang et al., 2016). 2) In bacterial infected-lung epithelial cells HMGN2 regulates pro-inflammatory cytokines, which have been shown to affect barrier function. For instance, TGF- $\beta$ mediates alcohol-induced decreases in lung epithelial barrier function while Granulocyte/macrophage colony-stimulating factor (GM-CSF) antagonizes TGF- $\beta$ to promote the barrier function (Overgaard et al., 2015). Additionally, Interferon- $\gamma$ and TNF- $\alpha$ have been shown to synergistically induce epithelial barrier dysfunction in intestinal duct (Wang et al., 2005). In summary, our data systemically investigated the overall effect of HMGN2 on bladder epithelial barrier function regulation and implies a potential cross-talk between 
transcellular and paracellular barrier defense against bacterial invasion.

\section{Conflict of interest statement}

The authors have declared no conflict of interest.

\section{Contributors}

Conception and design, or acquisition of data, or analysis and interpretation of data: H.T., J.M., F.Z., F.Z., N.H. and Y.W.; drafting the article or revising it critically for important intellectual content: Y.W., N.H., H.T., J.M., J.C. and J.L. All authors approved the final version to be published.

\section{Financial acknowledgements}

This work was supported by grants from the $\mathrm{Na}$ tional Natural Science Foundation of China (81470931, 31701098) and Sichuan University 985 Project-Science and Technology Innovation Platform for Novel Drug Development and Sichuan University Start-up Funds(2016 SCU11023, 2016SCU11021).

\section{REFERENCES}

Balkovetz DF, Katz J (2003) Bacterial invasion by a paracellular route: divide and conquer. Microbes Infect 5: 613-619. http://doi. org/10.1016/S1286-4579(03)00089-3

Billips BK, Forrestal SG, Rycyk MT, Johnson JR, Klumpp DJ, Schaeffer AJ (2007) Modulation of host innate immune response in the bladder by uropathogenic Escherichia coli. Infect Immun 75: 5353-5360. http://doi.org/10.1128/IAI.00922-07

Deng LX, Wu GX, Cao Y, Fan B, Gao X, Luo L, Huang N (2011) The chromosomal protein HMGN2 mediates lipopolysaccharideinduced expression of beta-defensins in A549 cells. FEBS J 278: 2152-2166. http://doi.org/10.1111/j.1742-4658.2011.08132.x

Deng T, Zhu ZI, Zhang S, Postnikov Y, Huang D, Horsch M, Bustin M (2015) Functional compensation among HMGN variants modulates the DNase I hypersensitive sites at enhancers. Genome Res 25: 1295-1308. http://doi.org/10.1101/gr.192229.115

Foxman B (1990) Recurring urinary tract infection: incidence and risk factors. Am J Public Health 80: 331-333. http://doi.org/10.2105/ AJPH.80.3.331

Guttman JA, Finlay BB (2009) Tight junctions as targets of infectious agents. Biochim Biophys Acta 1788: 832-841. http://doi.org/10.1016/j. bbamem.2008.10.028

Hashimoto J, Takahashi M, Saito A, Murata M, Kurimura Y, Nishitani C, Ariki S (2017) Surfactant protein A inhibits growth and adherence of uropathogenic Escherichia coli to protect the bladder from infection. J Immunol 198: 2898-2905. http://doi.org/10.4049/jimmunol.1502626
Hock R, Furusawa T, Ueda T, Bustin M (2007) HMG chromosomal proteins in development and disease. Trends Cell Biol 17: 72-79. http://doi.org/10.1016/j.tcb.2006.12.001

Hooton T. M, Besser R, Foxman B, Fritsche T. R, Nicolle L. E (2004) Acute uncomplicated cystitis in an era of increasing antibiotic resistance: a proposed approach to empirical therapy. Clin Infect Dis 39: 75-80. http://doi.org/10.1086/422145

Li Y, Lu M, Alvarez-Lugo L, Chen G, Chai TC (2017) Granulocytemacrophage colony-stimulating factor (GM-CSF) is released by female mouse bladder urothelial cells and expressed by the urothelium as an early response to lipopolysaccharides (LPS) Neurourol Urodyn 36: 1020-1025. http://doi.org/10.1002/nau.23057

Liu K, Wang X, Sha K, Zhang F, Xiong F, Wang X, Huang N (2017) Nuclear protein HMGN2 attenuates pyocyanin-induced oxidative stress via Nrf2 signaling and inhibits Pseudomonas aeruginosa internalization in A549 cells. Free Radic Biol Med 108: 404-417. http://doi. org/10.1016/j.freeradbiomed.2017.04.007

Ming L, Xiaoling P, Yan L, Lili W, Qi W, Xiyong Y, Ning H (2007) Purification of antimicrobial factors from human cervical mucus. Hum Reprod 22: 1810-1815. http://doi.org/10.1093/humrep/ dem128

Overgaard CE, Schlingmann B, Dorsainvil White S, Ward C, Fan X, Swarnakar S, Koval M (2015) The relative balance of GM-CSF and TGF-beta1 regulates lung epithelial barrier function. Am J Physiol Lung Cell Mol Physiol 308: L1212-L1223. http://doi.org/10.1152/ ajplung.00042.2014

Pitman RS, Blumberg RS (2000) First line of defense: the role of the intestinal epithelium as an active component of the mucosal immune system. J Gastroenterol 35: 805-814. http://doi.org/10.1007/ s005350070017

Schilling JD, Martin SM, Hung CS, Lorenz RG, Hultgren SJ (2003) Toll-like receptor 4 on stromal and hematopoietic cells mediates innate resistance to uropathogenic Escherichia coli. Proc Natl Acad Sci U S A 100: 4203-4208. http://doi.org/10.1073/pnas.0736473100

Teng Y, Miao J, Shen X, Yang X, Wang X, Ren L, Huang N (2016) The modulation of MiR-155 and MiR-23a manipulates Klebsiella pneumoniae adhesion on human pulmonary Epithelial cells via Integrin alpha5beta1 Signaling. Sci Rep 6: 31918. http://doi.org/10.1038/ srep31918

Wang F, Graham WV, Wang Y, Witkowski ED, Schwarz BT, Turner JR (2005) Interferon-gamma and tumor necrosis factor-alpha synergize to induce intestinal epithelial barrier dysfunction by up-regulating myosin light chain kinase expression. Am J Pathol 166: 409-419. http://doi.org/10.1016/S0002-9440(10)62264-X

Wang X, Li J, Chen S, Shen X, Yang X, Teng Y, Huang N (2016) Knockdown of HMGN2 increases the internalization of Klebsiella pneumoniae by respiratory epithelial cells through the regulation of alpha5beta1 integrin expression. Int J Mol Med 38: 737-746. http:// doi.org/10.3892/ijmm.2016.2690

Wu J, Miao Y, Abraham SN (2017) The multiple antibacterial activities of the bladder epithelium. Ann Transl Med 5: 35. http://doi. org $/ 10.21037 /$ atm.2016.12.71

Zhang S, Zhu I, Deng T, Furusawa T, Rochman M, Vacchio MS, Bustin M (2016) HMGN proteins modulate chromatin regulatory sites and gene expression during activation of naive B cells. Nucleic Acids Res 44: 7144-7158. http:/ /doi.org/10.1093/nar/gkw323 